\title{
Fasting Triglyceride is a Significant Risk Factor for Coronary Artery Disease in Middle-Aged Japanese Men
} Results From a 10-Year Cohort Study

\author{
Hiroki Satoh, MD; Tetsuo Nishino, MD*; \\ Kazuo Tomita, MD*; Hiroyuki Tsutsui, MD
}

\begin{abstract}
Background It has been well established that dyslipidemia is a significant risk factor for coronary artery disease (CAD), however, the association between fasting triglyceride (TG) and the occurrence of CAD is controversial. The objective of this study was to elucidate the relationship between fasting TG and CAD in middleaged Japanese men.

Methods and Results A cohort study of 6,966 middle-aged Japanese men (mean \pm SD: $46.6 \pm 5.2$ years) with a 10-year follow-up period was conducted to identify risk factors for the occurrence of CAD. One hundred and eleven cases of CAD were identified during the follow up. The Cox proportional hazard model was used to identify the independent risk factors for CAD. Adjustment was made for variables including age, body mass index, smoking habit, alcohol intake, duration of sleeping, systolic blood pressure, uric acid, total cholesterol, highdensity lipoprotein cholesterol, fasting plasma glucose, and TG. Fasting TG was identified as an independent risk factor for CAD. The adjusted hazard ratio of TG for CAD was 3.07 (95\% confidence interval: 1.01-9.35, $\mathrm{p}<0.05$ ). Additionally, a serum TG concentration level greater than $78 \mathrm{mg} / \mathrm{dl}$ was a significant risk for CAD.

Conclusions By using the long term follow-up data of the study of middle-aged Japanese men, fasting TG was identified to be a significant risk factor for CAD. (Circ J 2006; 70: 227-231)
\end{abstract}

Key Words: Coronary artery disease; High-density lipoprotein cholesterol; Total cholesterol; Triglyceride

A number of previous studies have established the relationship between high total cholesterol (TC) and low high-density lipoprotein cholesterol (HDL-C) and the development of coronary artery disease (CAD)!-6 In contrast, the association between triglyceride (TG) and the occurrence of CAD is controversial?,8 Previous studies using univariate analysis have demonstrated that TG is a significant risk factor for CAD?-12 In contrast, other studies have shown that this relationship was not statistically significant after adjusting with TC and HDL-C by using the multivariate regression analyses $!^{13-15}$ However, these studies have been performed in Western countries and might not be directly applicable to the Japanese population because the incidence of CAD has been reported to be lower in Japan compared to that in Europe and the USA ${ }^{16}$ Although one study reported that non-fasting TG could predict the occurrence of CAD in the Japanese population, ${ }^{17}$ the relationship between fasting TG and the risk for CAD has not been established. Therefore, the purpose of the present study was to elucidate the relationship between fasting TG and the development of CAD among 6,966

(Received October 5, 2005; revised manuscript received December 5, 2005; accepted December 22, 2005)

Department of Cardiovasucular Medicine, Hokkaido University Graduate School of Medicine, *The Health Management Center, NTT East Japan Sapporo Hospital, Sapporo, Japan

Mailing address: Hiroki Satoh, MD, Department of Cardiovasucular Medicine, Hokkaido University Graduate School of Medicine, Kita 15, Nishi 7, Kita-ku, Sapporo 060-8638, Japan. E-mail: h-satoh@ imb.me-h.ne.jp middle-aged Japanese men using the database obtained from the cohort study with the follow-up period of 10 years.

\section{Methods}

Study Subjects

The study subjects included 7,403 male workers, aged 33-59 years old, in a company in Hokkaido, Japan, from 1995 to 2005. 215 subjects who had not had aphysical examination at baseline and 26 subjects who had already been diagnosed as having CAD were excluded from the present study. 196 subjects who left the company during the follow-up period were also excluded. Therefore, a total of 6,966 subjects were included in the present study conducted in 1995. During the follow-up period of 10 years, 81 subjects who died a non-CAD death and 8 who died because of CAD were included in the present study. The study protocol was approved by the ethical committee of the NTT East Japan Sapporo Hospital, Sapporo, Japan.

\section{Data Collection}

At baseline, blood samples were obtained from subjects in the morning after an overnight fast. A blood sample was obtained from the antecubital vein and the serum was separated. After precipitation by heparin-manganese, TC and HDL-C were measured by using the phosphotungstate method. TG and uric acid (UA) were measured enzymatically. Fasting plasma glucose (FPG) was enzymatically determined by using the hexokinase method. Baseline blood pressure (BP) was measured by a trained nurse using 
Table 1 Baseline Characteristics for Subjects With and Without Coronary Artery Disease During a 10-Year Follow up

\begin{tabular}{lccc}
\hline \hline Variable & $\begin{array}{c}C A D \\
(n=111)\end{array}$ & $\begin{array}{c}\text { No CAD } \\
(n=6,855)\end{array}$ & p value \\
\hline Age (years) & $46.6 \pm 5.2$ & $46.7 \pm 5.2$ & 0.48 \\
Body mass index & $24.9 \pm 3.2$ & $23.4 \pm 2.9$ & $<0.01$ \\
Smokers $(\%)$ & 82.9 & 62.3 & $<0.01$ \\
Alcohol $(\mathrm{g} /$ day) & $22.08 \pm 20.7$ & $26.38 \pm 22.7$ & $<0.05$ \\
Duration of sleep & 3.3 & 2.7 & 0.44 \\
$\quad($ less than $5 \mathrm{~h})(\%)$ & $132.2 \pm 18.3$ & $126.6 \pm 17.5$ & $<0.01$ \\
Systolic BP $(\mathrm{mmHg})$ & $219.3 \pm 32.0$ & $201.9 \pm 32.7$ & $<0.01$ \\
TC $(\mathrm{mg} / \mathrm{dl})$ & $157(113-207)$ & $110(78-159)$ & $<0.01$ \\
TG $(\mathrm{mg} / \mathrm{dl})$ & $43.6 \pm 12.2$ & $53.5 \pm 16.3$ & $<0.01$ \\
HDL-C $(\mathrm{mg} / \mathrm{dl})$ & $108.0 \pm 40.3$ & $97.4 \pm 22.4$ & $<0.01$ \\
$F P G(\mathrm{mg} / \mathrm{dl})$ & $5.9 \pm 1.3$ & $5.9 \pm 1.4$ & 0.49 \\
UA $(\mathrm{mg} / \mathrm{dl})$ & & & \\
\hline
\end{tabular}

Data are presented as mean $\pm S D$. TG was expressed as a median and interquartile range due to its skewed distribution. $p<0.05$ considered to be significant.

$C A D$, coronary artery disease; $B P$, blood pressure; $T C$, total cholesterol; $T G$, triglyceride;HDL-C, high-density lipoprotein cholesterol; FPG, fasting plasma glucose; UA, uric acid;

a standard mercury sphygmomanometer with the participant in the sitting position after at least a 5-min rest. Bodyweight and height were measured in the morning while the subjects were in a fasting state. Body mass index (BMI) was calculated as bodyweight $(\mathrm{kg})$ divided by squared height $\left(\mathrm{m}^{2}\right)$. Smoking habit, alcohol intake, and duration of sleep were determined by using a self-reported questionnaire. Subjects who had never smoked and ex-smokers were classified as 'non-smokers'. Subjects were divided into 2 groups, that is, one group who had more than $5 \mathrm{~h}$ of sleep and the other group who had less than $5 \mathrm{~h}$ of sleep.

\section{Outcome Measures}

During the follow-up period of 10 years (mean \pm SD: $9.5 \pm 0.8$ years), any sick leave taken by the subjects was immediately reported to the company's health management center. The occurrence of CAD was identified annually between 1995 and 2005 after an examination of all the details of a subject's clinical chart.

CAD was identified as acute myocardial infarction and angina pectoris. The criteria for CAD were modified from World Health Organization Expert Committee 18 Angina pectoris was defined as repeated episodes of chest pain during effort and usually disappearing rapidly after the cessation of effort or on use of sublingual nitroglycerin. Additionally, subjects with angina pectoris had coronary stenosis of greater than $75 \%$ of the luminal diameter by coronary angiography 19 Acute myocardial infarction was defined by the presence of at least 2 of the following criteria: a history of prolonged discomfort or anginal equivalent, ECG changes consistent with ischemia or necrosis, and elevated cardiac enzymes.

\section{Statistical Analysis}

Continuous variables were expressed as mean $\pm \mathrm{SD} ; \mathrm{TG}$ was described as a median (and interquartile range) for variables with a skewed distribution, and smoking habit and duration of sleep were described as a percentage. The differences of variables between the 2 groups were examined by using the Student's unpaired t-test for approximately normal distributed variables, or by the Wilcoxon rank-sum test for TG, and by the Fisher's exact test for the
Table 2 HRs for Coronary Artery Disease With CIs for Risk Factors With Adjustment for All Variables

\begin{tabular}{lccc}
\hline \hline Variable & Adjusted HR & $95 \%$ CI & p value \\
\hline Age & 1.00 & $0.96-1.03$ & 0.81 \\
Body mass index & 1.05 & $0.99-1.12$ & 0.12 \\
Smoking & 5.59 & $2.85-10.96$ & $<0.001$ \\
Alcohol & 0.92 & $0.88-0.96$ & 0.05 \\
Duration of sleep & 1.00 & $0.97-1.02$ & 0.87 \\
Systolic BP & 1.01 & $1.02-1.25$ & 0.03 \\
TC & 1.14 & $1.07-1.21$ & $<0.001$ \\
log TG & 3.07 & $1.01-9.35$ & $<0.05$ \\
HDL-C & 0.80 & $0.73-0.89$ & $<0.001$ \\
FPG & 1.05 & $1.01-1.10$ & 0.03 \\
UA & 0.86 & $0.74-1.00$ & 0.05 \\
\hline
\end{tabular}

$p<0.05$ is considered to be significant.

HR, hazard ratio; CI, confidence interval; BP, blood pressure; TC, total cholesterol; TG, triglyceride; HDL-C, high-density lipoprotein cholesterol; FPG, fasting plasma glucose; UA, uric acid.

proportion of smoking habit and duration of sleep. The Cox proportional hazard model was used to examine the relationship between risk factors and CAD and to access the unadjusted and adjusted hazard ratio (HR) of events. The principle model included candidate variables for age (years), smoking habit (non and current smokers), alcohol consumption (g/day), duration of sleep (less and more than $5 \mathrm{~h})$, BMI, systolic BP $(\mathrm{mmHg}), \mathrm{TC}(\mathrm{mg} / \mathrm{dl})$, HDL-C $(\mathrm{mg} / \mathrm{dl}), \log \mathrm{TG}, \mathrm{FPG}(\mathrm{mg} / \mathrm{dl})$, and UA $(\mathrm{mg} / \mathrm{dl})$. Age and BMI were indicated by one additional increase. Systolic BP was indicated by an additional $10 \mathrm{mmHg}$ increase. Both TC and FPG were indicated by an additional $10 \mathrm{mg} / \mathrm{dl}$ increase, and HDL-C was indicated by an additional $5 \mathrm{mg} / \mathrm{dl}$ increase. TG was calculated as a log-transformed value. A p value less than 0.05 was considered to indicate statistical significance. All statistical analyses were performed by using the SPSS statistical package for Windows version 12.0 (Chicago, IL, USA) ${ }^{20}$

\section{Results}

During the follow-up period of 10 years, 111 subjects had CAD; 74 subjects had acute myocardial infarction and 37 subjects had angina pectoris. The age at baseline was $46.6 \pm 5.2$ years (ranging from 33 to 55 years), and that at onset of CAD was $51.6 \pm 5.7$ years (ranging from 36 to 59 years).

The baseline characteristics of the subjects with and without CAD are shown in Table 1. Subjects with CAD were more likely smokers and less likely drinkers. They had a greater BMI, systolic BP, TC, TG, and FPG levels, and a lower HDL-C level. There were no significant differences in other variables such as age, duration of sleep and UA between these 2 groups of subjects.

By using the Cox proportional hazard models, smoking habit, systolic BP, TC, TG, FPG and low HDL-C were identified as significant independent risk factors for CAD (Table 2). The HR of CAD adjusting for risk factors with smoking habit was 5.59 (95\% confidence interval (CI): $2.85-10.96, \mathrm{p}<0.001)$, with a $10 \mathrm{mmHg}$ increase in systolic BP was 1.01 (95\% CI: $1.02-1.25, \mathrm{p}<0.05)$, with a $10 \mathrm{mg} / \mathrm{dl}$ increase in TC was 1.14 (95\% CI: 1.07-1.21, $\mathrm{p}<0.001)$, with a one increase in log TG was 3.07 (95\% CI: 1.01-9.35, $\mathrm{p}<0.05)$, with a $5 \mathrm{mg} / \mathrm{dl}$ increase in HDL-C was 0.80 (95\% CI: $0.73-0.89$, p<0.001), and with a $10 \mathrm{mg} / \mathrm{dl}$ increase in FPG was 1.05 (95\% CI: 1.01-1.10, p<0.05). 
Table 3 HRs for Coronary Artery Disease With CIs for TG Levels

\begin{tabular}{cccc}
\hline \hline Model & Hazard ratio & $95 \%$ CI & $p$ value \\
\hline 1 & 23.74 & $10.10-55.81$ & $<0.001$ \\
2 & 10.16 & $3.81-27.08$ & $<0.001$ \\
3 & 6.63 & $2.34-18.76$ & 0.001 \\
4 & 3.07 & $1.01-9.35$ & $<0.05$
\end{tabular}

Model 1, unadjusted; Model 2, adjusted for age, BMI, smoking, alcohol, duration of sleep, systolic BP, FPG, UA, and TC; Model 3, adjusted for age, $B M I$, smoking, alcohol, duration of sleep, systolic BP, FPG, UA, and HDLC; Model 4, adjusted for age, BMI, smoking, alcohol, duration of sleeping, systolic $B P, F P G, U A, T C$, and $H D L-C$. $p<0.05$ is considered to be significant.

$H R$, hazard ratio; CI, confidence interval; TG, triglyceride; BMI, body mass index; BP, blood pressure; FPG, fasting plasma glucose; UA, uric acid; TC, total cholesterol; $H D L-C$, high-density lipoprotein cholesterol.

By using the Cox proportional hazard models, TG was identified as a significant independent risk factor for CAD (Table 3). The unadjusted HR of CAD for TG was 23.74 (95\% CI: 10.10-55.81, p<0.001). TG was an independent risk factor for CAD even after the adjustment with such unfounding variables such as TC and HDL-C. The adjusted HR of CAD was 3.07 (95\% CI: 1.01-9.35, p<0.05).

HRs for CAD were further assessed within the quartile levels of TG ranging $\leq 78,79-110,111-161$, and $\geq 162 \mathrm{mg} / \mathrm{dl}$ (Table 4). There was a dose-response relationship between TG and the risk of CAD. Comparing the lowest to the second lowest quartile level of TG, the unadjusted HR was 7.22 (95\% Cl: 2.15-24.20, p<0.01). After adjusting for TC, HDL-C, and other variables, the adjusted HR of CAD was 4.13 (95\% Cl: 1.22-14.00, $\mathrm{p}<0.05)$.

HDL-C values in the quartile levels of TG are shown in Fig 1. There was a negative relationship between the quartile levels of TG and HDL-C values. The quartile levels of TG from the lowest to highest had HDL-C values $($ mean \pm SD) of $63.8 \pm 17.6,55.6 \pm 14.8,50.1 \pm 13.5$, and $43.8 \pm 11.6 \mathrm{mg} / \mathrm{dl}$, respectively.

HRs for CAD were further assessed by the combination of TG and HDL-C levels (Table 5). We divided these into 3 groups: the low TG and high HDL-C (group 1), the high TG and high HDL-C (group 2), and the high TG and low HDL-C (group 3). If group 1 was considered to be a reference, the adjusted HR of CAD in group 2 and 3 was 5.52 (95\% CI: $1.71-17.85, \mathrm{p}<0.01)$ and 12.59 (95\% CI: 3.82 $41.46, \mathrm{p}<0.001)$, respectively.
Table 4 HRs for Coronary Artery Disease With CIs According to Quartile Levels of TG

\begin{tabular}{|c|c|c|c|c|}
\hline $\begin{array}{l}\text { Quartile }(Q) \text { of } \\
\text { triglycerides }\end{array}$ & $Q 1$ & $Q 2$ & $Q 3$ & $Q 4$ \\
\hline \multicolumn{3}{|l|}{ Model 1} & 30.6 & 47.7 \\
\hline$H R$ & 1.0 & 7.22 & 11.44 & 18.75 \\
\hline $\begin{array}{l}95 \% C I \\
p \text { value }\end{array}$ & & $\begin{array}{c}2.15-24.20 \\
<0.01\end{array}$ & $\begin{array}{l}3.51-37.24 \\
<0.01\end{array}$ & $\begin{array}{c}5.85-60.03 \\
<0.01\end{array}$ \\
\hline \multicolumn{5}{|l|}{ Model 2} \\
\hline $\begin{array}{l}\text { HR } \\
\quad 95 \% C I \\
\quad \text { value }\end{array}$ & 1.0 & $\begin{array}{c}5.57 \\
1.65-18.75 \\
<0.01\end{array}$ & $\begin{array}{c}7.41 \\
2.24-24.46 \\
<0.01\end{array}$ & $\begin{array}{c}10.31 \\
3.12-34.05 \\
<0.001\end{array}$ \\
\hline \multicolumn{5}{|l|}{ Model 3} \\
\hline$H R$ & 1.0 & 6.78 & 9.24 & 11.38 \\
\hline $\begin{array}{l}95 \% C I \\
\text { p value }\end{array}$ & & $\begin{array}{c}1.49-16.98 \\
<0.05\end{array}$ & $\begin{array}{l}1.94-21.31 \\
<0.05\end{array}$ & $\begin{array}{c}2.40-26.96 \\
<0.05\end{array}$ \\
\hline \multicolumn{5}{|l|}{ Model 4} \\
\hline$H R$ & 1.0 & 4.13 & 4.44 & 4.87 \\
\hline $95 \% C I$ & & $1.22-14.00$ & $1.32-14.93$ & $1.42-16.69$ \\
\hline$p$ value & & $<0.05$ & $<0.05$ & $<0.05$ \\
\hline
\end{tabular}

$\overline{Q 1}, 2,3$, and 4 are $T G$ levels $\leq 78,79-110,111-161$, and $\geq 162 \mathrm{mg} / \mathrm{dl}$, respectively.

Models 1, 2, 3, and 4 are shown in Table 3. $p<0.05$ is considered to be significant.

$H R$, hazard ratio; $C I$, confidence interval; TG, triglyceride.

Table 5 HRs for Coronary Artery Disease With CIs According to the Combination of TG and HDL-C

\begin{tabular}{lccc}
\hline \hline & $\begin{array}{c}\text { Low TG and } \\
\text { High HDL-C } \\
\text { group }\end{array}$ & $\begin{array}{c}\text { High TG and } \\
\text { High HDL-C } \\
\text { group }\end{array}$ & $\begin{array}{c}\text { High TG and } \\
\text { Low HDL-C } \\
\text { group }\end{array}$ \\
\hline Unadjusted HR & 1.00 & 6.21 & 19.62 \\
95\%CI & & $1.45-26.57$ & $4.83-79.69$ \\
p value & & $<0.05$ & $<0.001$ \\
Adjusted HR & 1.00 & 5.52 & 12.59 \\
95\%CI & & $1.71-17.85$ & $3.82-41.46$ \\
p value & & $<0.01$ & $<0.001$ \\
\hline
\end{tabular}

TG level was divided into a high or low group, which was more or less than $78 \mathrm{mg} / \mathrm{dl}$.

HDL-C level was divided into a high or low group, which was more or less than $40 \mathrm{mg} / \mathrm{dl}$.

$p<0.05$ is considered to be significant.

$H R$, hazard ratio; CI, confidence interval; TG, triglyceride; HDL-C, highdensity lipoprotein cholesterol.

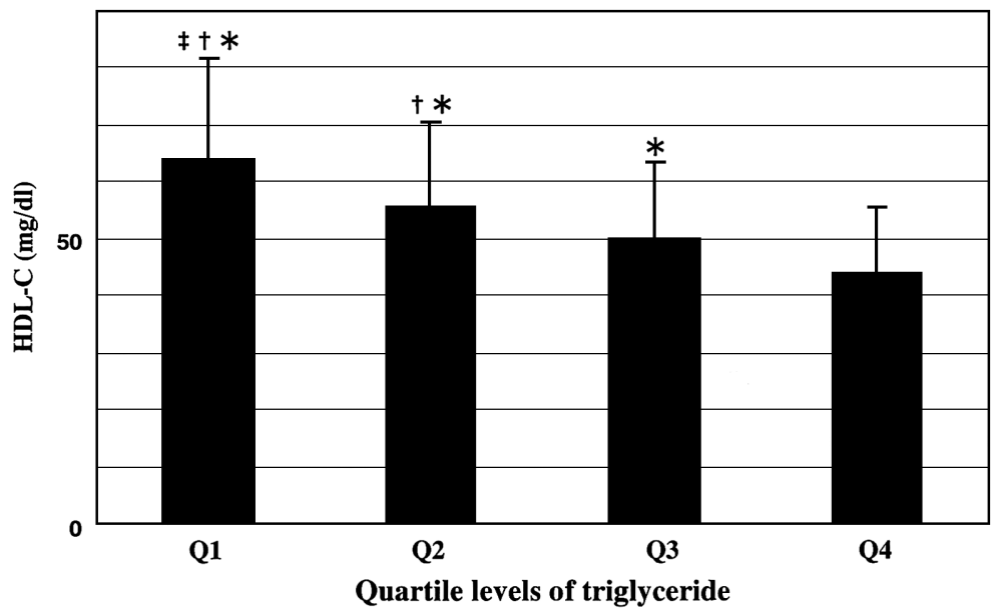

Fig 1. High-density lipoprotein cholesterol (HDL-C) values in the quartile $(\mathrm{Q})$ levels of triglyceride (TG; Q1, 2,3 and 4 ) are TG levels $\leq 78,79-110,111-161$, and $\geq 162 \mathrm{mg} / \mathrm{dl}$, respectively. ${ }^{*} \mathrm{p}<0.01$ compared with $\mathrm{Q} 4$, $* * \mathrm{p}<0.01$ compared with $\mathrm{Q} 3, * * * \mathrm{p}<0.01$ compared with Q2. 


\section{Discussion}

The present study indicated that fasting TG is significantly associated with the development of CAD in middle-aged Japanese men. A serum TG concentration level greater than $78 \mathrm{mg} / \mathrm{dl}$ is a significant risk for CAD. To our knowledge, this is the first epidemiological study to demonstrate a significant relationship between fasting TG and CAD among Japanese men.

The relationship between dyslipidemia and CAD risk has been well established3,21,22 Previous studies have demonstrated that high TC increases the risk for CAD and a $1 \%$ reduction in serum $\mathrm{TC}$ concentration reduces $\mathrm{CAD}$ risk by $2 \%$ ! The present study has shown that a $10 \mathrm{mg} / \mathrm{dl}$ increase in TC increases CAD risk by $14 \%$. Even though hyperlipidemia is an established risk factor for CAD, approximately $40 \%$ of CAD patients had a TC level below the normal range, and most of these patients had low levels of HDL-C, regardless of the TG levels 23 Recently, the International Diabetes Federation showed that other lipoproteins such as HDL-C and TG could be the potential therapeutic targets? Low levels of HDL-C is a well-established risk factor for the development of CAD. Previous studies demonstrated that a $1 \%$ increase in HDL-C is associated with a $2-3 \%$ reduction in the risk of CAD5 The present study similarly demonstrated that a decreased of CAD risk was $20 \%$ by a $5 \mathrm{mg} / \mathrm{dl}$ increase of HDL-C.

Previous studies have reported inconsistent results regarding the association between TG and CAD?-17 The Copenhagen Male Study, which followed 2,906 white men over 8 years, found that fasting TG was independently associated with the incidence of CAD. However, when adjusted for HDL-C and low-density lipoprotein (LDL) cholesterol levels, TG did not increase the risk for CAD? In most studies, the relationship between $\mathrm{TG}$ and $\mathrm{CAD}$ risk is not statistically significant, especially in men9,25 The present study demonstrated that fasting TG has a dosedependent relationship with the development of CAD, with adjusting for HDL-C, TC and other risk factors. A previous study demonstrated a close correlation of remnant-like particle (RLP)-cholesterol levels with TG levels in the Japanese population.26 RLP-cholesterol contributed to atherogenesis by directly affecting the vascular, endothelial and smooth muscle cells. ${ }^{27}$ This is because fasting TG might be an independent risk factor for CAD in our population.

Additionally, the analysis of quartile levels of TG indicated that $\mathrm{TG}$ greater than $78 \mathrm{mg} / \mathrm{dl}$ was a significant risk for causing CAD. By comparing 2 groups that are greater or less than $78 \mathrm{mg} / \mathrm{dl}$, the adjusted HR of CAD was 4.18 (95\% Cl: 1.35-14.24, $\mathrm{p}=0.014$ ) (data not shown) and the same result was indicated.

Both TG and HDL-C are inversely correlated and mechanistically linked means of lipid transfer activities ${ }^{28}$ In our population, the increase of serum TG concentration affected the decrease of serum HDL-C concentration. These results demonstrated that subjects who had higher levels of TG could have another risk factor such as low HDL-C, which might have more risk for CAD occurrence. The present study demonstrated that subjects who had a high TG level and a low HDL-C level had a significantly higher risk of having CAD than those who had a high TG level and a normal HDL-C level.

Hypertriglycemia is associated with increased concentrations of factor VII and plasminogen activator inhibitor, which may accelerate thrombotic processes 29 Hyperglyc- emia is also closely associated with small, dense LDL, which has increased entry into and retention in the arterial wall because of a low affinity for LDL receptors and susceptibility to oxidation; this is considered to be more atherogenic than larger LDL particles. ${ }^{29-33}$ However, it is difficult to assess LDL particle size directly. These results have indicated that the measurement of TG is considered to be important to predict the risk for the development of CAD.

\section{Study Limitations}

The present study subjects included only males who were aged between 33 and 55 years. Therefore, we need to be cautious to extend the present study results to the general population. Recently, metabolic syndrome was a significant risk factor for the development of CAD, and abdominal obesity and insulin resistance were crucial problems. These risk factors were not investigated in the present study and further examinations using these variables were required. However, the present study design enables us to enrol and perform the long-term follow-up studies in many subjects.

In conclusion, the present study demonstrated that fasting TG is an independent risk factor for CAD among middle-aged Japanese men.

\section{References}

1. Expert Panel on Detection, Evaluation and Treatment of High Blood Cholesterol in Adults. Executive Summary of the third report of the National Cholesterol Education Program (NCEP) expert panel on detection, evaluation, and treatment of high blood cholesterol in adults (Adult Treatment Panel III). JAMA 2001; 285: 2486-2497.

2. WHO Expert Committee on Prevention of Coronary Heart Disease. Prevention of coronary disease. Geneva, Switzerland: World Health Organization, 1982 (World Health Organization technical report series 678).

3. Castelli WP, Garrison RJ, Wilson PW, Abbott RD, Kalousdian S, Kannel WB. Incidence of CAD and lipoprotein cholesterol levels: The Framingham Study. JAMA 1986; 256: 2835-2838.

4. WHO; Reducing risks, promoting healthy life. The World Health Report 2002

5. Boden WE. High-density lipoprotein cholesterol as an independent risk factor in cardiovascular disease: Assessing the data from Framingham to the Veterans Affairs High-Density Lipoprotein Intervention Trail. Am J Cardiol 2000; 86: 19L-22L.

6. Rubin HB, Robin SJ, Collins D, Anderson JW, Elam MB, Faas FH, et al. Distribution of lipids in 8,500 men with coronary artery disease. Am J Cardiol 1995; 75: 1196-1201.

7. Szapary PO, Rader DJ. The triglyceride-high-density lipoprotein axis: An important target of therapy? Am Heart J 2004; 148: 211-221.

8. Austin MA. Plasma triglyceride as a risk factor for coronary heart disease: The epidemiologic evidence and beyond. Am J Epidemiol 1989; 129: 249-259.

9. Jeppesen J, Hein HO, Suadicani P, Suadicani P, Gyntelberg F. Triglyceride concentration and ischemic heart disease: An eight-year follow-up in the Copenhagen Male Study. Circulation 1998; 97: 1029-1036.

10. Menotti A, Scanga M, Morisi G. Serum triglycerides in the prediction of coronary heart disease (an Italian experience). Am J Cardiol 1994; 73: 29-32.

11. Stampfer MJ, Krasuss RM, Ma J, Blanche PJ, Holl LG, Sacks FM, et al. A prespective study of triglyceride level, low-density lipoprotein particle diameter, and risk of myocardial infarction. JAMA 1996; 276: $882-888$

12. Bengtsson C, Bjorkelund C, Lapidus L, Lissner L. Associations of serum lipid concentrations and obesity with mortality in women: 20 year follow-up of participants in prospective population study in Gothenburg, Sweden. BMJ 1993; 307: 1385-1388.

13. Wilson PWF, Larson MG, Castelli WP. Triglycerides, HDL cholesterol and coronary heart disease: A Framingham update on their interrelations. Can J Cardiol 1994; 10(Suppl B): 5B-9B.

14. Criqui MH, Heiss G, Cohn R, Cowan LD, Suchindran CM, Bangdiwala S. Plasma triglyceride level and mortality from coronary heart disease. N Engl J Med 1993; 328: 1220-1225. 
15. Assmann G, Schulte H. Relation of high-density lipoprotein cholesterol and triglycerides to incidence of atherosclerotic coronary artery disease (the PROCAM experience). Am J Cardiol 1992; 70: $733-$ 737.

16. Sekikawa A, Satoh T, Hayakawa T, Ueshima H, Kuller LH. Coronary heart disease mortality among men aged $35-44$ years by prefecture in Japan in 1995-1999 compared with among white men aged 35-44 by state in the United States in 1995-1998: Vital statistics in recent birth cohort. Jpn Circ J 2001; 65: 887-892.

17. Iso H, Naito $Y$, Sato S, Kitamura A, Okamura T, Sankai T, et al. Serum triglycerides and risk of coronary heart disease among Japanese men and women. Am J Epidemiol 2001; 153: 490-499.

18. WHO Expert Committee. Arterial Hypertension and Ischemic Heart Disease, Preventive Aspects. Geneva: WHO Technical Report Series No 231, 1962.

19. AHA Committee Report: A reporting system on patient evaluated for coronary artery disease. Circulation 1975; 51: 5-34.

20. SPSS Incorporation. 2003 SPSS Base 12.05J User's Guide. Chicago, IL; SPSS Inc, 2003.

21. National Cholesterol Education Program. Second report of the Expert Panel on Detection, Education, and Treatment of High Blood Cholesterol in Adults (Adults Treatment Panel II). Circulation 1994; 89: $1333-1445$.

22. Nakaya N, Kita T, Mabuchi H, Matsuzaki M, Matsuzawa Y, Oikawa $\mathrm{S}$, et al. Large-scale cohort study on the relationship between serum lipid concentration and risk of cerebrovascular disease under lowdose simvastatin in Japanese patients with hypercholesterolemia: Sub-analysis of the Japan Lipid Intervention Trial (J-LIT). Circ J 2005; 69: 1016-1021.

23. Hokanson JE, Austin MA. Plasma triglyceride level is a risk factor for cardiovascular disease independent of high-density lipoprotein cholesterol level: A meta-analysis of population-based prospective studies. J Cardiovas Risk 1996; 3: 213-219.

24. Athyros VG, Ganotakis ES, Elisaf M, Mikhailidis DP. The prevalence of the metabolic syndrome using the National Cholesterol Edu- cation Program and International Diabetes Federarion definitions. Curr Med Res Opin 2005; 21: 1157-1160.

25. Avins AL, Neuhaus JM. Do triglycerides provide meaningful information about heart disease risk? Arch Intern Med 2000; 160: $1937-$ 1944.

26. Arai H, Yamamoto A, Matsuzawa Y, Saito Y, Yamada N, Oikawa S, et al. Serum lipid survey and its recent trend in the general Japanese population in 2000. J Atheroscler Thromb 2005; 12: 98-106.

27. Kawakami A, Yoshida M. Remnant lipoprotein and atherogenesis. J Atheroscler Thromb 2005; 12: 73-76.

28. Fournier N, Atger V, Cogny A, Vedie B, Girel P, Simon A, et al. Analysis of the relationship between triglyceride and HDL-phospholipid concentrations: Consequences on the efflux capacity of serum in the Fu5AH system. Atherosclerosis 2001; 157: 315-323.

29. Krauss RM. Heterogeneity of plasma low density lipoproteins and atherosclerosis risk. Curr Opin Lipidol 1994; 5: 339-349.

30. de Graaf J, Hak-Lemmers HLM, Hectors MPC, Demacker PNM, Hendriks JCM, Stalenhof AFH. Enhanced susceptibility to in vitro oxidation of the dense low density lipoprotein subfraction in healty subjects. Arterioscler Thromb 1991; 11: 298-306.

31. Galeano NF, AI-Haiseri M, Keyserman F, Rumsey SC, Deckelbaum RJ. Small dense low density lipoprotein has increased affinity for LDL receptor-independent cell surface binding sites: A potential mechanism for increased atherogenicity. J Lipid Res 1998; 39: $1263-1273$.

32. Kondo A, Muranaka Y, Ohta I, Notsu K, Manabe M, Kotani K, et al. Relationship between triglyceride concentrations and LDL size evaluated by malondialdehyde-modified LDL. Clin Chem 2001; 47: $893-900$.

33. Tsunoda F, Koba S, Hirano T, Ban Y, Iso Y, Suzuki H, et al. Association between small dense low-density lipoprotein and postprandial accumulation of triglyceride-rich remnant-like particles in normotriglyceridemic patients with myocardial infarction. Circ J 2004; 68: $1165-1172$. 